\title{
Serum Level of IL-4 Predicts Response to Topical Immunotherapy with Diphenylcyclopropenone (DPCP) in Alopecia Areata
}

Yugang Gong ${ }^{1,2}$, Ying Zhao ${ }^{1}$, Xiaoting Zhang ${ }^{1}$, Shiling Qi ${ }^{1}$, Shuifeng Li $^{1}$, Yanting Ye ${ }^{1}$, Jian Yang ${ }^{1}$, Sillani Caulloo ${ }^{1}$, Kevin J. McElwee ${ }^{3}$, *Xingqi Zhang ${ }^{1}$.

${ }^{1}$ Department of Dermatology, The First Affiliated Hospital, Sun Yat-sen University, 58 Zhongshan Road II, Guangzhou, Guangdong 510080, People's Republic of China.

${ }^{2}$ Institute of Dermatology and Venereology, Sichuan Academy of Medical Sciences \& Sichuan Provincial People's Hospital, No. 32, Western 2nd Section, Chengdu, Sichuan 610032, People's Republic of China.

${ }^{3}$ Centre for Skin Science, University of Bradford, Bradford, UK.

*Correspondence: Xingqi Zhang, M.D., Ph.D.

Department of Dermatology, The First Affiliated Hospital, Sun Yat-sen University, 58

Zhongshan Road II, Guangzhou, Guangdong 510080, People's Republic of China.

Funding source: National Natural Science Foundation of China (81573066); Natural Science Foundation of Guangdong Province (2014A030313098)

Conflicts of Interest: Kevin McElwee is Chief Scientific Officer for Replicel Life Sciences Inc. All other authors state no conflict of interest.

Word count for the text (excluding references, figures, and tables): 3448

The number of references: 42

The number of figures: 2

The number of tables: 2

Key Words: Alopecia areata; diphenylcyclopropenone; therapeutic effect; prognostic biomarkers 


\section{Abstract}

Background: This study investigated predictors of response to topical diphenylyclopropenone (DPCP) immunotherapy in patients with alopecia areata (AA). Objective: To identify predictors of response, or resistance, to treatment for AA through clinical observations and serum tests. Methods: 84 AA patients were treated with DPCP. Serum cytokine levels were measured in 33 AA patients pre- and post-treatment, and in 18 healthy controls, using ELISA assays. Results: $56.1 \%$ of patients responded to DPCP with satisfactory hair regrowth; the response rate was negatively correlated with hair loss extent. Before DPCP treatment, higher serum IFN- $\gamma$ and IL-12 cytokine levels were observed in AA patients compared to healthy controls. Non-responders to DPCP had significantly elevated serum IL-4 pre-treatment (3.07 fold higher) and lower IL-12 levels compared with responders. After DPCP treatment, non-responders had persistently high IL-4, increased IL-12, negligible decrease in IFN- $\gamma$ and decreased IL-10. Post-treatment DPCP responders exhibited significantly decreased IFN- $\gamma$ and IL-12, and increased IL-4 and IL-10. Development of adverse side-effects was significantly associated with higher pre-treatment serum IgE levels. Limitations: a small number of subjects were evaluated. Conclusions: Potentially, elevated pre-treatment serum levels of IL-4 and IL-12 can be used as unfavorable and favorable predictors of DPCP therapeutic effect, respectively. In addition, pre-treatment elevated serum total IgE may predict increased risk for severe adverse side-effects to DPCP application. Whether serum cytokine expression levels can be used as predictors of response to other forms of treatment is unknown, but it may warrant investigation in the development of personalized treatments for AA. 


\section{Introduction}

Alopecia areata (AA) is a common non-scarring inflammatory hair disorder, typically characterized by sudden onset of hair loss in the form of round or oval patches with a clear margin. So far, the etiopathogenesis of AA is not fully understood. ${ }^{[1-6]}$ Studies suggest that in AA pathogenesis, a disturbance of T helper cell type 1 (Th1) and type 2 (Th2) immune equilibrium can occur. Elevation of Th1 related cytokines in response to various factors may change the immunologic privilege of hair follicles, enhancing CD8+ T lymphocyte cytotoxicity and natural killer cell (NK) activity, subsequently inducing follicular cell apoptosis, which contributes to hair loss in AA. ${ }^{[1-3,6-8]}$ Current AA treatment modalities mainly include topical and systemic corticosteroids, T cell inhibitors, and topical hair follicle growth stimulants. ${ }^{[9-11]}$ Severe cases of AA can require long-term systemic corticosteroids or immunosuppressive agents, which are not as effective as in mild cases, and may have significant adverse side-effects.

Squaric acid dibutylester and diphenylcyclopropenone (DPCP) have been used extensively as contact sensitizers for AA treatment. ${ }^{[12-17]}$ DPCP is a non-mutagenic chemical substance with a high sensitization potency, which does not react with other contact allergens; therefore it is a common agent used internationally in sensitization treatment of extensive AA. ${ }^{[14-15,17-18]}$ DPCP treatment has been found to have an efficacy rate ranging from $5 \%$ to $85 \%$, with a relapse rate ranging from $10.6 \%$ to $68.9 \%$ in AA patients. ${ }^{[14-15,18-23]}$

Although DPCP is considered as a good option to treat extensive AA, not all patients respond. A predictive indicator of response to DPCP is highly desirable for efficient treatment provision and to limit negative patient experiences. In this study, we present our experience with DPCP treatment in patients with severe types of AA, as well as the results of clinical and laboratory data analysis to understand the mechanism of action, and to identify biomarkers that predict the effectiveness of, and tolerance to, DPCP through convenient serum tests. 


\section{Materials and Methods}

\subsection{Clinical Data}

A total of 84 patients with AA were enrolled into DPCP treatment at the Hair Clinic, Department of Dermatology, First Affiliated Hospital of Sun Yat-sen University, in the years 2009 to 2012. Detailed clinical data for these patients were recorded. Inclusion criteria included chronic AA (disease duration more than 12 months), lack of response to other treatments, and more than $25 \%$ scalp area affected. Cessation of any topical or systemic AA therapy for at least six weeks was required prior to the start of DPCP treatment. 50 of 84 patients were observed via dermoscopy, all of which exhibited the dermoscopic criteria of AA. The characteristic dermoscopic signs presented in the hair loss area included visible hair follicle ostia, yellow dots, cadaverized hairs (black dots), exclamation mark hairs, short vellus hair and newly-grown short hairs. The other 34 AA patients were not observed via dermoscopy as dermoscopy was not available when the patients started their treatment (before the year 2010). In these patients, the diagnosis of AA was made mainly by history, clinical presentation, and examinations. Exclusion criteria included $<25 \%$ scalp area affected, pregnancy, significant cardiovascular disease, or serious medical illness. Informed written consent was obtained from all patients. All the procedures in this study were approved by The Ethics Committee of the First Affiliated Hospital of Sun Yat-sen University (No. 200990) and conform to the principles of the Declaration of Helsinki.

\subsection{Therapeutic Regime}

Diphenylyclopropenone (diphencyprone) in acetone, at serial dilutions of $0.001 \%$, $0.01 \%, 0.05 \%, 0.1 \%, 0.25 \%, 0.5 \%, 1.0 \%$, and $2.0 \%$, was used in ascending order to seek the 
optimal concentration in treating AA patients. At the first visit, each patient was sensitized with $2 \%$ DPCP on an area of $2 \mathrm{~cm}$ in diameter behind the ear. After 2 weeks, progressively higher concentrations were applied to half of the scalp at weekly intervals until an acceptable response was achieved; ranging from $0.001 \%$ to $2 \%$ according to each patient's response. The other half of the scalp was not treated and was used as an intra-control to identify hair growth responses specific to DPCP. Once hair growth was established on one side, the other side was also treated. Patients were instructed to avoid direct sun exposure and to wash DPCP out only at $48 \mathrm{~h}$ after each application.

\subsection{Efficacy Evaluation}

Response of patients was evaluated by global photography and dermoscopy with a non-contact polarized dermoscope (Dermlite, DL3 model, 3Gen US, USA). The area of hair loss in the patients was evaluated using the SALT score method. ${ }^{[24]}$ Hair regrowth was evaluated by the MacDonald-Hull and Norris grading system, i.e., grade I, vellus hair; grade II, sparse pigmented terminal hair; grade III, terminal hair regrowth with patches of alopecia; grade IV, growth of terminal hair over all of the treated scalp area. ${ }^{[25]}$ Responders were defined as having grade III and grade IV hair regrowth scores, and non-responders as having grade II and grade I hair regrowth scores as well as no hair regrowth within 6 months after starting treatment. Relapse after achievement of hair regrowth was defined as when new hair loss affected more than $25 \%$ of the hair re-growth observed during DPCP therapy. Data was collected including the duration of DPCP treatment, concentration that elicited a good response, presence and type of side-effects, and relapse after completing treatment.

\subsection{Laboratory Tests}


Complete blood count, serum IgE levels, thyroid antibodies (thyroid globulin antibody, TG-AB; thyroid peroxidase antibodies, TPO-AB), thyroid gland function (T3, T4, TSH) and immunological laboratory tests such as autoantibodies (ANA, ds-DNA, AHA, AnuA, DNP) were monitored pre- and post-treatment using immunoblotting, a semi-quantitative latex slide test, and radial immunodiffusion assays (R\&D Systems, Shanghai, China) according to manufacturers' protocols.

\subsection{Cytokine Expression Analysis}

Serum cytokine levels were measured in 21 patients in the responder group and 12 patients in the non-responder group both pre- and post-treatment, and in 18 healthy controls, using ELISA assays (R\&D Systems, Shanghai, China). Serum cytokine levels of interferon gamma (IFN- $\gamma$ ), interleukin (IL)-2, IL-4, IL-5, IL-10, IL-12, IL-17, IL-18, and IL-22 were measured.

\subsection{Statistical Analysis}

All analyses were performed using the software programs SPSS 13.0 (SPSS Inc, Chicago, IL). One-way analysis of variance (ANOVA) tests were conducted for continuous variables with normal distribution (One-way ANOVA). Non-parametric tests were used to evaluate differences between continuous variables which did not conform to the normal distribution (Shapiro-Wilk W test). $\chi^{2}$ test was used to show differences between categorical variables. According to the type of quantitative data, paired $t$ test or Wilcoxon matchedsamples signed rank sum test were respectively used to evaluate differences between continuous variables pre- and post-treatment. Statistical significance was set at $\mathrm{P}=0.05$. $\mathrm{P}$ values less than 0.05 were considered significant. 


\section{Results}

\subsection{Clinical Data}

In this group of 84 patients there were 38 males and 46 females, where age at onset of therapy with DPCP ranged from 5 to 63 years (mean 27.1 12.7 years), and duration of hair loss ranged from 0.2 to 25 years (mean $6.0 \pm 5.4$ years). The area of hair loss in these 84 patients ranged $25-100 \%$ (mean $82.3 \% \pm 25.0 \%$ ), with 7 patients $(8.3 \%)$ in Grade I $(25-49 \%$ scalp involvement), 20 patients (23.8\%) in Grade II (50-74\% scalp involvement), 11 patients (13.1\%) in Grade III (75-99\% scalp involvement) and 46 patients (54.8\%) in Grade IV as alopecia universalis (AT) and alopecia totalis (AU) affected subjects.

\subsection{Response to DPCP Therapy}

A total of 57 patients received DPCP treatments for more than 6 months, with the time long enough to evaluate the response to DPCP treatment (Figure S1). Of these, 32 patients had satisfactory hair regrowth (responders), and the overall rate of response was $56.1 \%$ (32/57), in which, 75.0\% (3/4) were Grade I (25-49\% scalp involvement), 73.3\% (11/15) were Grade II (50-74\% scalp involvement), 85.7\% (6/7) were Grade III (75-99\% scalp involvement) and 38.7\% (12/31) were Grade IV (AT and AU). Statistical analysis showed a negative correlation existed between response rate and hair loss area $(\chi 2=8.386, \mathrm{P}=0.004)$, but no significant association was found between response to treatment and the final concentration of DPCP applied, duration of AA, age, gender, atopic history (personal history of any atopic symptoms), nail involvement and other clinical features (data not shown).

Patients were divided into four groups according to their therapeutic response, i.e., responders (32/84), non-responders (25/84), withdrawal due to severe side-effects (7/84) and voluntary withdrawal (20/84). The clinical and laboratory characteristics of the four treatment 
groups are presented (Table 1). In the responder group, 19 cases were in grade III and 13 cases were in grade IV according to the MacDonald-Hull and Norris grading system, ${ }^{[22]}$ therapeutic effect was seen as early as the 6th week to the 22nd week, with an average of 10.6 \pm 4.4 weeks. Twenty-seven patients withdrew for varied reasons, such as being unable to attend the clinic for weekly therapy (14 cases) and non-responsiveness to DPCP (6 cases). In addition, therapy was discontinued in 7 patients due to severe side-effects including development of vitiligo in one patient and intolerance to DPCP presented as systemic contact dermatitis in 6 patients.

\subsection{Treatment Reaction and Side-Effects}

About $93 \%$ of patients (78/84) experienced one or more side-effects during treatment. Local dermatitis of the scalp in the DPCP contact area was the common finding in most of patients $(70 / 78,89.7 \%)$. Local lymphadenopathy in the draining area was another common side-effect $(38 / 78,48.7 \%)$. Other side-effects included scalp hyperpigmentation (32/78, $41.0 \%)$, systemic contact dermatitis $(11 / 78,14.1 \%)$, scalp hypopigmentation $(4 / 78,5.1 \%)$, hyperpyrexia $(1 / 78,1.3 \%)$ and vitiligo $(1 / 78,1.3 \%)$. Apart from vitiligo, hyperpyrexia and systemic contact dermatitis, these side-effects in $87.7 \%$ (71/78) patients resolved after topical corticosteroid application, or with the use of systemic antihistamines, or after stopping the treatment for one week, but they did not lead to discontinuation of therapy.

\subsection{Relapse}

A relapse rate of $40.6 \%$ (13/32) was observed. Relapse happened 4 to 65 weeks after establishment of hair regrowth and these patients were followed up for a period ranging from 9 to 40 months after stopping the treatment. Of these patients, 4 progressed to $\mathrm{AU}$, who started a new treatment program with DPCP therapy to achieve 
excellent responses again. The remaining 9 patients lost $25 \%$ to $50 \%$ of re-grown hair, who did not start a new treatment. There was no significant association between the final concentration of DPCP applied and the relapse rate.

\subsection{Dermoscopic Features}

Dermoscopy was performed in 50 patients before and after DPCP treatment, including 22 responders, 17 non-responders, 2 in the severe side-effects group, and 9 in the voluntary withdrawal group (Figure S2). The incidence of yellow dots, broken hairs, cadaverized hairs (black dots), and exclamation mark hairs before treatment were 54.0\% (27/50), 62.0\% (31/50), $62.0 \%(31 / 50)$ and $30.0 \%(15 / 50)$, respectively (Table 2). The incidence of broken hairs was higher in the responder group $(77.3 \%)$ than in the non-responder group $(41.2 \%)\left(\chi^{2}=5.28\right.$, $\mathrm{P}=0.022$ ). With treatment, the average time of disappearance of yellow dots, broken hairs, cadaverized hairs (black dots) and exclamation mark hairs in the responder group was 17.1, 14.6, 12.2 and 9.1 weeks respectively, but no correlation was found with effectiveness of treatment. In the non-responder group, besides 3 cases, not all the typical dermoscopic signs of AA had disappeared in the same patient.

\subsection{Laboratory Tests}

Prior to treatment, elevated serum levels of T3 and T4 were found in 10 patients and 1 patient, respectively. Serum levels of TSH were found elevated in 3 patients, but decreased in 1 patient. Autoantibodies to the thyroid gland, TG-Ab and TPO-Ab were found positive in 17 and 9 patients, respectively. Percentages and cell counts of peripheral eosinophils were found increased in 7 and 2 patients, respectively. ANA was found positive in 8 patients and increased IgE levels presented in 19 patients (Table 1). Comparison of the laboratory data among the responder, non-responder, and withdrawal due to severe side-effects groups, 
revealed no significant difference except that pre-treatment serum $\operatorname{IgE}$ levels were significantly higher in the withdrawal due to severe side-effects group than in the responder group $(\chi 2=6.68, \mathrm{P}=0.010)$ and the non-responder group $(\chi 2=5.80, \mathrm{P}=0.016)$. No significant differences were observed between responder and non-responder groups. Elevated IgE levels also revealed no significant difference between the patients with and without atopic history.

\subsection{Cytokine Profiles}

Serum cytokine levels were measured in 21 patients in the responder group and 12 patients in the non-responder group, both pre- and post-treatment, who completed treatments for more than 6 months, with the time long enough to evaluate the response to DPCP treatment, and also in 18 healthy controls. Before DPCP treatment in all AA patients, serum levels of IFN- $\gamma$ and IL-12 were higher while IL-5 was lower than that in the healthy control group. No statistical significance was found on comparison of IL-2, IL-4, IL-10, IL-17, IL-18 and IL-22 levels of AA patients to the normal control group (data not shown for the latter two cytokines). AA patients were divided further into responder and non-responder groups according to their response to DPCP treatment. In the responder group, serum levels of IFN- $\gamma$, IL-12 were higher, and IL-5 was lower, than controls while IL-2, IL-4, IL-10 and IL-17 were similar to that of controls. In contrast, in the non-responder group, serum levels of IFN- $\gamma$, IL-4, IL-12 and IL-17 were higher while IL-5 was lower than normal controls (Figure 1). In direct comparison with the responder group, significantly higher IL-4 and lower IL-12 serum levels were found in the non-responder group pre-treatment (Figure 2). Interestingly, in DPCP nonresponders, the IL-4 level was 3.07 and 3.10 fold higher compared to responders and controls respectively, whereas the IL-12 level was 0.75 fold lower than that of responders.

After DPCP treatment, there was a marked decrease in serum IFN- $\gamma$, IL-12 and an increase in IL-5, IL-10 and IL-17 levels in all AA patients as a group. Among them, IFN- $\gamma$ 
was restored to a level similar to healthy controls, but IL-12, IL-4, IL-5 and IL-17 were higher than normal controls. Although IL-10 was higher in controls than AA patients prior to treatment, after the treatment it was similar to that of controls. However, there was an interesting discrepancy in cytokine profiles between responder and non-responder groups. In the responder group, marked decreased serum IFN- $\gamma$ and IL-12 levels were noted posttreatment as compared to pre-treatment levels, and restored to the level of normal controls. However, increased IL-4, IL-5, IL-10 and IL-17 serum levels were found in responders posttreatment when compared to pre-treatment levels as well as to controls.

In contrast, in the non-responder group, the decrease in IFN- $\gamma$ serum level was only mild, and no significant difference was found between pre- and post-treatment levels, both being higher than that in the control group. An increase in post-treatment serum IL-12 level was found as compared to pre-treatment, rather than a decrease as seen in the responder group. Increased IL-5 and IL-17 serum levels were found in the non-responder group when compared to pre-treatment levels as well as to controls. IL-4 level remained high, and no difference was noted in serum IL-4 and IL-10 pre- and post-treatment (Figure 1). When comparing between responder and non-responder groups after the treatment, higher IL-4, IL-12, IFN- $\gamma$ and lower IL-10 serum levels were found in the non-responder group (Figure 2).

\section{Discussion}

The treatment of severe AA can still be a difficult task; development of new and improved treatments for extensive AA is an area of active investigation. ${ }^{[26]}$ Topical immunotherapy offers long-term therapeutic hope for patients with this distressing condition. At present, DPCP is considered to be an effective and safe therapeutic method for AA, more precisely for severe cases of AA with a relatively low rate of adverse-effects. ${ }^{[18-23]}$ The effectiveness of topical immunotherapy with AA has been demonstrated in several reports, 
although the response rate varied greatly from $5 \%$ to $85 \% .^{[14-15,18-23]}$ The discrepancy of response rates may be due to the type and severity of the AA, the number of patients in the clinical study, and different methods of assessing clinical efficacy.

In this study, we present our experience from the use of DPCP in the treatment of patients with severe AA in China. The response rate to DPCP with satisfactory hair regrowth was around 56\%; in concordance with other previous studies using similar inclusion criteria and efficacy evaluation methodology. ${ }^{[18-23]}$ The most common iatrogenic effect is local contact dermatitis. ${ }^{[18-23]}$ Certain clinical features, such as history of atopy and autoimmune diseases, duration of AA, nail involvement, thyroid antibodies, etc., have been associated with AA disease severity in previous studies, ${ }^{[27-28]}$ but were not observed in our patients. The only variable significantly correlated with the prognosis was the hair loss area extent of AA.

Pre-treatment serum IgE levels were significantly higher in the withdrawal due to severe side-effects group than in the responders group and non-responders group. Therefore, serum IgE level could be used as a prognostic marker to indicate DPCP intolerance. The welldocumented high relapse rate reported in previous medical literature was also observed in our patients $(40.6 \%)$. However, continuous treatment for maintenance of hair growth is generally recommended and still effective for patients with relapse. ${ }^{[25,29]}$ Similarly, our relapsed cases that continued or restarted on DPCP therapy achieved excellent responses again.

It is generally accepted that AA is a T lymphocyte mediated inflammatory disease on a multigene background, likely triggered by external factors. ${ }^{[1-6]}$ Remission in AA occurs with a decrease in Th1 cytokines and relative increase in Th2 cytokines which prompts or enables anagen, clinically perceived as hair regrowth. ${ }^{[1-3,9,30-35]}$ In our study, prior to treatment, in all AA patients as a group, serum levels of Th1 type cytokines IFN- $\gamma$ and IL-12 were higher, while the Th2 type cytokine IL-5 was lower. These results support the notion of Th1 prevalence in the etiopathogenesis of AA. 
The exact mechanism of action of the immune-modulating effect in DPCP is unclear, but to date it is generally assumed that it acts on different pathways to inhibit inflammation in alopecic areas which is achieved mainly by suppression of inflammatory cells, such as CD4+ and CD8+ T cells, restoration of equilibrium between Th1 and Th2 responses, or involvement of regulatory T cells. ${ }^{[36-42]}$ In our study, post-treatment levels of serum Th1 type cytokines IFN- $\gamma$ and IL-12 were significantly decreased, and levels of serum Th2 type cytokines IL-4 and IL-10 were increased in the responder group, which was different from the non-responder group. Thus, AA patients with a predominant Th1 profile are good candidates for DPCP treatment, using IL-12 as an indicator, as it was higher in responders than in non-responders prior to treatment. The new cytokine milieu in response to DPCP treatment is likely crucial in promoting or enabling entry of hair follicles into an anagen stage leading to hair regrowth. However, any further change in the new cytokine balance can also induce the relapse of hair loss.

In contrast, in the DPCP non-responder group, both Th1 and Th2 cytokines were higher than that of normal controls prior to DPCP treatment. Most notably, the serum IL-4 level was 3 fold higher than that observed in both controls and responders before DPCP treatment. IL-4 also remained high after the failed treatment, suggesting a persistent enhancement of Th2 activity. Moreover, non-responders had no significant decrease in IFN- $\gamma$ serum levels, an increase of IL-12, and a lower level of IL-10 after DPCP treatment indicating a failure to inhibit Th1 activity.

Therefore, a pre-existing high Th2 cytokine profile can apparently counteract the therapeutic immune response elicited by DPCP treatment, perhaps resulting in failure to inhibit the pathogenic Th1 cytokine activity underlying AA. Thus, elevated pre-treatment serum IL-4 levels in AA patients could be used as a negative prognostic marker of DPCP therapeutic efficacy. The data indicate AA patients with a high Th2 cytokine profile are not 
good candidates for DPCP treatment, even with a coexisting high Th1 cytokine profile. The functional significance of elevated IL-4 in AA is unclear, but it suggests there is also a role for Th2 type cytokines in AA pathogenesis in at least some patients, as indicated by other

research. ${ }^{[42]}$ Increased serum IL-5 and IL-17 levels were also found both in responder and non-responder groups after treatment when compared to pre-treatment levels, as well as to controls; possibly a response caused by the DPCP induced contact dermatitis. Their role in modulating the pathogenic Th1 activity of AA is unclear.

In conclusion, the response rate to DPCP of Chinese patients with AA was similar to other previous studies involving other ethnic groups and correlated negatively with extent of hair loss. Elevated pre-treatment serum total IgE levels can be used as a predictor of DPCP intolerance and elevated pre-treatment serum levels of IL-4 and IL-12 can be used as unfavorable and favorable predictors of response to DPCP treatment, respectively. Taken together, this study provides evidence that the mode of DPCP action may restore of the immunological balance by counteracting the pathogenic dominant Th1 reaction of AA.

\section{Acknowledgments}

This work is supported by the National Natural Science Foundation of China (81573066) and Natural Science Foundation of Guangdong Province (2014A030313098) to Xingqi Zhang.

\section{Author contributions}

Xingqi Zhang initiated and supervised the research. Yugang Gong performed the research and analysed data and wrote the manuscript. Ying Zhao performed the research and analysed data. Xiaoting Zhang, Shiling Qi, Shuifeng Li, Yanting Ye, Jian Yang, Wenna Chen, Sillani Caulloo performed the research. Kevin J. McElwee advised on the research and edited the 
manuscript. All the listed authors have reviewed the manuscript and have approved this final version for publication. 


\section{References}

[1] M. Bertolini, A. Gilhar, R. Paus, Exp Dermatol. 2012, 21, 477.

[2] K.J. McElwee, D.J. Tobin, J.-C. Bystryn, L.E. King Jr, J.P. Sundberg, Exp Dermatol. 2007, 8,371 .

[3] T. Ito, Y. Tokura, Exp Dermatol. 2014, 23, 787.

[4] T. Ito, K.C. Meyer, N. Ito, R. Paus, Curr Dir Autoimmun. 2008, 10, 27.

[5] K. J. McElwee A. Gilhar D. J. Tobin Y. Ramot J. P. Sundberg M. Nakamura M. Bertolini S. Inui Y. Tokura L. E. King B. Duque - Estrada A. Tosti A. Keren S. Itami Y. Shoenfeld A. Zlotogorski R. Paus, Exp Dermatol. 2013, 22, 609.

[6] A.J. Mcdonagh, J.A. Snowden, C. Stierle, K. Elliott, A.G. Messenger, Br J Dermatol. 1993, 129, 250.

[7] R. Paus R, A. Slominski, B.M. Czarnetzki, Yale J Biol Med. 1993, 66, 541.

[8] A. Gilhar, M. Landau, B. Assy, Y. Ullmann, R. Shalaginov, S. Serafimovich, R.S. Kalish, Clinical Immunol. 2003, 106, 181.

[9] J. Fuentes-Duculan, N. Gulati, K.M. Bonifacio, N. Kunjravia, X. Zheng, M. SuárezFariñas, A. Shemer, E. Guttman-Yassky, J.G. Krueger. Exp Dermatol. 2016, 25, 282.

[10] S. Garg, A.G. Messenger, Semin Cutan Med Surg. 2009, 28, 15.

[11] M. Miteva, A. Tosti, Expert Opin Pharmacother. 2012, 13, 1271.

[12] L.A. Daman, E.W. Rosenberg, L. Drake, Arch Dermatol. 1978, 114, 1036.

[13] P.C. Case, A.J. Mitchell, N.A. Swanson, E.E. Vanderveen, C.N. Ellis, C.N. Headington, J Am Acad Dermatol. 1984, 10, 447.

[14] R. Happle, B.M. Hausen, L. Wiesner-Menzel, Acta Derm Venereol. 1983, 63, 49.

[15] S.M. Hull, W.J. Cunliffe, Br J Dermatol. 1991, 124, 212. 
[16] C.K. Rokhsar, J.L. Shupack, J.J. Vafai, K. Washenik, J Am Acad Dermatol, 1998, 39, 751.

[17] K.F. Mose, M. Burton, M. Thomassen, F. Andersen, T.A. Kruse, Q. Tan, L. Skov, M.A. Røpke, T. Litman, O. Clemmensen, B.W. Kristensen, P.S. Friedmann, K.E. Andersen, Exp Dermatol. 2017, 26, 926.

[18] S.P. MacDonald-Hull, M.L. Wood, P.E. Hutchinson, M. Sladden, A.G. Messenger, Br J Dermatol. 2003, 149, 692.

[19] B.M. El-Zawahry, D.A. Bassiouny, A. Khella, N.S. Zaki, J Eur Acad Dermatol Venereol. 2010, 24, 264.

[20] K. Chiang, N. Atanaskova Mesinkovska, A. Amoretti, M.P Piliang, A. Kyei, W.F. Bergfeld, J Am Acad Dermatol. 2014, 71, 595.

[21] N.M. Luk, L.S. Chiu, K.C. Lee, C.T. Chau, V. Lee, M. Chang, Y.K. Lam, H.C. Lee, J Eur Acad Dermatol Venereol. 2013, 27, e400.

[22] M.C. Wiseman, J. Shapiro, N. Macdonald, H. Lui, Arch Dermatol. 2001, 137, 1063.

[23] G. Avgerinou, S. Gregoriou, D. Rigopoulos, A. Stratigos, D. Kalogeromitros, A. Katsambas, J Eur Acad Dermatol Venereol. 2008, 22, 320.

[24] E.A. Olsen, Dermatol Ther. 2011, 24, 311.

[25] S.M. Hull, J.F. Norris, Br J Dermatol. 1988, 119, 367.

[26] J. Sun, K.A. Silva, K.J. McElwee, L.E. King Jr, J.P. Sundberg. Exp Dermatol. 2008, $17,793$.

[27] F.L. Xiao FL, D.Q. Ye, S. Yang, F.S. Zhou, S.M. Zhou, Y.G. Zhu, Y.H. Liang, Y.Q. Ren, X.J. Zhang, J Eur Acad Dermatol Venereol. 2006, 20, 1207.

[28] C. Goh, M. Finkel, P.J. Christos, A.A. Sinha, J Eur Acad Dermatol Venereol. 2006, 20, 1055.

[29] R. Happle, Arch Dermatol. 2002, 138, 112. 
[30] S. Gregoriou, D. Papafragkaki, G. Kontochristopoulos, E. Rallis, D. Kalogeromitros, D. Rigopoulos, Mediators Inflamm. 2010, 2010, 928030.

[31] A. Gilhar, M. Landau, B. Assy, R. Shalaginov, S. Serafimovich, R.S. Kalish, Arch Dermatol. 2002, 138, 916.

[32] N. Barahmani, A. Lopez, D. Babu, M. Hernandez, S.E. Donley, M. Duvic, Clin Exp Dermatol. 2010, 35, 409.

[33] E.A. Attia, S.D. El-Shennawy, A. Sefin, Dermatol Res Pract. 2010, 2010, 503587.

[34] K. Katagiri, S. Arakawa, Y. Hatano, Arch Dermatol Res. 2007, 298, 397.

[35] I.J. Crane, J.V. Forrester, Crit Rev Immunol. 2005, 25, 75.

[36] V. Herbst, M. Zöller, S. Kissling, E. Wenzel, N. Stutz, P. Freyschmidt-Paul, Eur J Dermatol. 2006, 16, 537.

[37] P. Gupta, P. Freyschmidt-Paul, M. Vitacolonna, S. Kissling, S. Hummel, D. Hildebrand, R. Marhaba, M. Zöller, J Invest Dermatol. 2006, 126, 1559.

[38] M. Zöller, K.J. McElwee, M. Vitacolonna, R. Hoffman, Exp Dermatol. 2004, 13, 435.

[39] R. Hoffmann, E. Wenzel, A. Huth, P. van der Steen, M. Schäufele, H.P. Henninger, R. Happle, J Invest Dermatol. 1994, 103, 530.

[40] H. Ujiie, Exp Dermatol. 2018, in press.

[41] N. Gulati, M. Suarez-Farinas, J. Fuentes-Duculan, P. Gilleaudeau, M. SullivanWhalen, J. Correa da Rosa, I. Cueto, H. Mitsui, J.G. Krueger, J Invest Dermatol. 2014 , $134,2531$.

[42] T, Czarnowicki, H.Y. He, H.C. Wen, P.W. Hashim, J.K. Nia, K. Malik, Y. Estrada, G.W. Kimmel, M. Taliercio, J.G. Krueger, E. Guttman-Yassky. Allergy. 2018, 73, 713. 


\section{Figures}
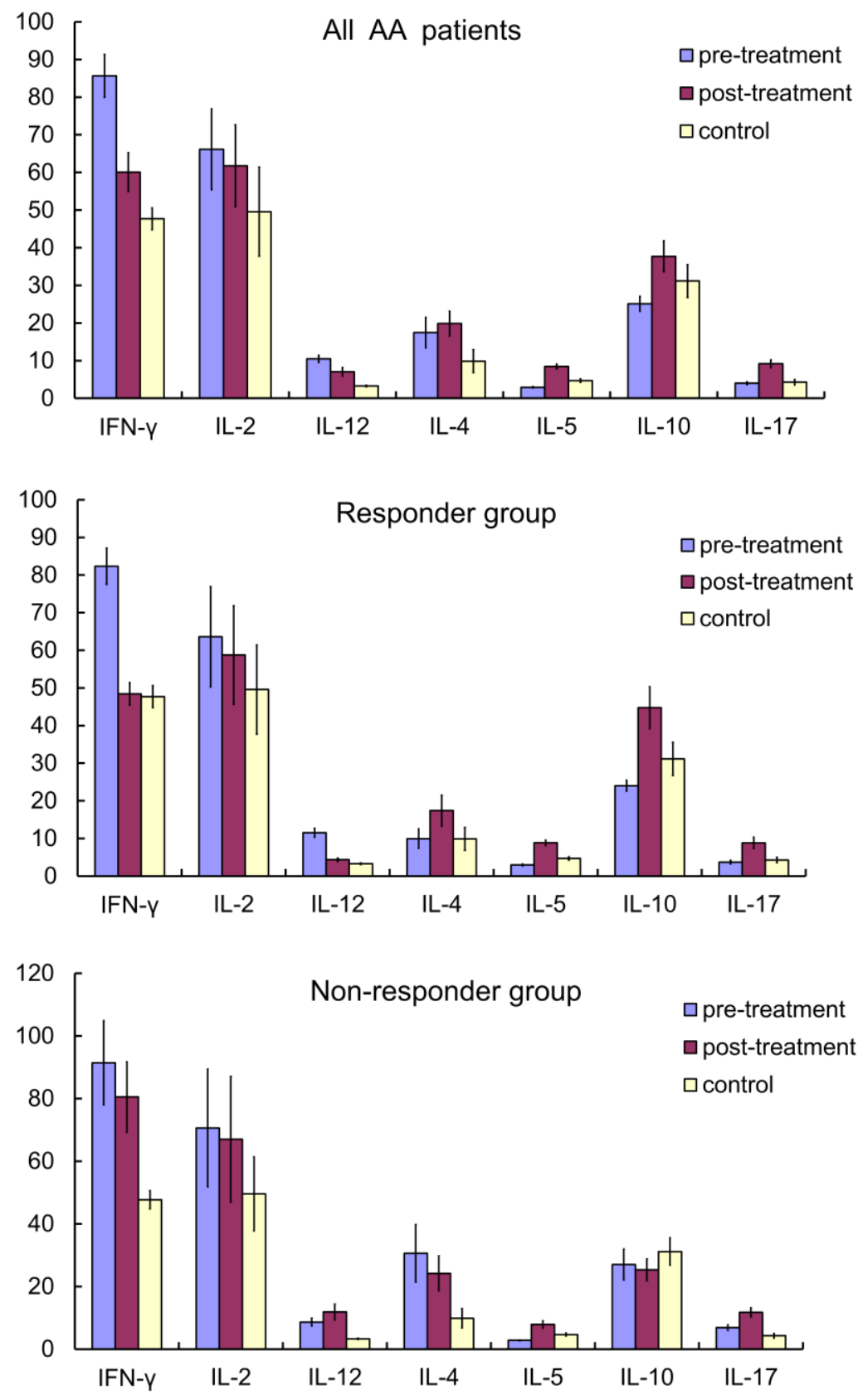

Figure 1. Serum cytokine profiles of all AA patients

Serum cytokine profiles of all AA patients pre- and post-topical immunotherapy (A), and as compared to healthy controls. AA patients are evaluated separately as responders to DPCP (B) and non-responders to DPCP (C) (unit: $\mathrm{pg} / \mathrm{ml}, * P<0.05$, $* * P<0.01$ ). 

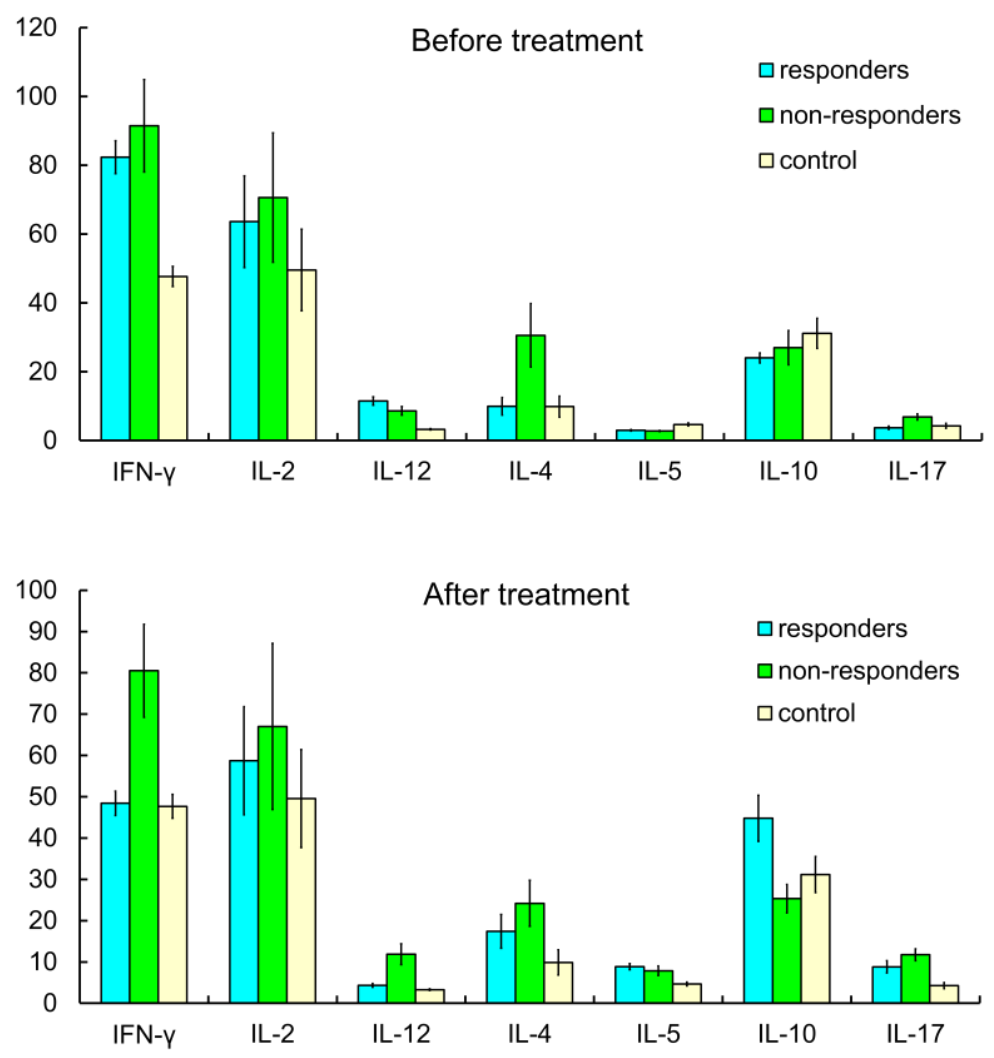

Figure 2. Serum cytokine profiles of AA patients in DPCP responders and nonresponders

Serum cytokine profiles of AA patients in the responder group compared to the non-responder group and healthy controls prior to treatment (A) and post-treatment (B) (unit: $\mathrm{pg} / \mathrm{ml},{ }^{*} P<0.05$, $* * P<0.01)$ 
Tables

Table 1. Clinical and laboratory data in four treatment groups

\begin{tabular}{|c|c|c|c|c|c|c|c|c|c|c|c|}
\hline Groups & $\begin{array}{c}\text { Patients' } \\
\text { age } \\
\text { (years) }\end{array}$ & $\begin{array}{c}\text { Duration } \\
\text { of } \\
\text { disease } \\
\text { (years) }\end{array}$ & Hair loss area & $\begin{array}{c}\text { Family } \\
\text { history of } \\
\text { AA }\end{array}$ & $\begin{array}{l}\text { Atopic } \\
\text { history }\end{array}$ & $\begin{array}{c}\text { TG-AB } \\
\text { increase }\end{array}$ & $\begin{array}{l}\text { TPO-AB } \\
\text { increase }\end{array}$ & $\begin{array}{c}\text { EO\# } \\
\text { increase }\end{array}$ & $\begin{array}{c}\mathrm{EO} \% \\
\text { increase }\end{array}$ & $\begin{array}{c}\text { ANA } \\
\text { increase }\end{array}$ & $\begin{array}{c}\operatorname{IgE} \\
\text { increase }\end{array}$ \\
\hline $\begin{array}{l}\text { Responder } \\
\text { group }\end{array}$ & $\begin{array}{c}28.3 \pm \\
11.5\end{array}$ & $6.4 \pm 1.2$ & $76.4 \% \pm 27.1 \%$ & $\begin{array}{c}4 / 32 \\
(12.5 \%)\end{array}$ & $\begin{array}{c}3 / 32 \\
(9.4 \%)\end{array}$ & $\begin{array}{c}9 / 32 \\
(28.1 \%)\end{array}$ & $\begin{array}{c}2 / 32 \\
(6.3 \%)\end{array}$ & $\begin{array}{c}1 / 32 \\
(3.1 \%)\end{array}$ & $\begin{array}{c}3 / 32 \\
(9.4 \%)\end{array}$ & $\begin{array}{c}4 / 32 \\
(12.5 \%)\end{array}$ & $\begin{array}{c}5 / 32 \\
(15.6 \%)\end{array}$ \\
\hline $\begin{array}{l}\text { Non- } \\
\text { responder } \\
\text { group }\end{array}$ & $\begin{array}{c}25.8 \pm \\
11.7\end{array}$ & $5.7 \pm 4.1$ & $90.0 \% \pm 19.8 \%$ & $\begin{array}{c}3 / 25 \\
12.0 \%)\end{array}$ & $\begin{array}{c}3 / 25 \\
(12.0 \%)\end{array}$ & $\begin{array}{c}4 / 25 \\
(16.0 \%)\end{array}$ & $\begin{array}{c}3 / 25 \\
(12.0 \%)\end{array}$ & $\begin{array}{c}1 / 25 \\
(4.0 \%)\end{array}$ & $\begin{array}{c}2 / 25 \\
(8.0 \%)\end{array}$ & $\begin{array}{c}1 / 25 \\
4.0 \%)\end{array}$ & $\begin{array}{c}4 / 25 \\
(16 \%)\end{array}$ \\
\hline $\begin{array}{l}\text { Severe } \\
\text { side-effect } \\
\text { group }\end{array}$ & $\begin{array}{c}27.0 \pm \\
16.8\end{array}$ & $8.3 \pm 5.2$ & $82.9 \% \pm 24.3 \%$ & $0 / 7$ & $\begin{array}{c}1 / 7 \\
(14.3 \%)\end{array}$ & $0 / 7$ & $0 / 7$ & $0 / 7$ & $0 / 7$ & $0 / 7$ & $\begin{array}{c}5 / 7 \\
(71.4 \%)^{*}\end{array}$ \\
\hline $\begin{array}{l}\text { Voluntary } \\
\text { withdrawal } \\
\text { group }\end{array}$ & $\begin{array}{c}27.0 \pm \\
15.0\end{array}$ & $4.8 \pm 4.7$ & $81.8 \% \pm 26.4 \%$ & $\begin{array}{c}2 / 20 \\
10.0 \%)\end{array}$ & $\begin{array}{c}3 / 20 \\
(15.0 \%)\end{array}$ & $\begin{array}{c}4 / 20 \\
(20.0 \%)\end{array}$ & $\begin{array}{c}4 / 20 \\
(20.0 \%)\end{array}$ & $0 / 20$ & $\begin{array}{c}2 / 20 \\
(10.0 \%)\end{array}$ & $\begin{array}{c}3 / 20 \\
(15.0 \%)\end{array}$ & $\begin{array}{c}5 / 20 \\
(25.0 \%)\end{array}$ \\
\hline $\mathrm{P}$ value & 0.903 & 0.494 & 0.066 & $\begin{array}{c}0.628 \\
(\chi 2=1.74)\end{array}$ & $\begin{array}{c}0.937 \\
(\chi 2=0.42)\end{array}$ & $\begin{array}{c}0.204 \\
(\chi 2=4.60)\end{array}$ & $\begin{array}{c}0.275 \\
(\chi 2=3.88)\end{array}$ & $\begin{array}{c}0.658 \\
(\chi 2=1.61)\end{array}$ & $\begin{array}{c}0.721 \\
\left(\chi^{2}=1.33\right)\end{array}$ & $\begin{array}{c}0.332 \\
\left(\chi^{2}=3.42\right)\end{array}$ & $\begin{array}{c}\mathbf{0 . 0 2 6} \\
(\chi 2=9.23)\end{array}$ \\
\hline
\end{tabular}


Table 2. Dermoscopic features in four treatment groups

\begin{tabular}{lcccc} 
Groups & Yellow dots & Broken hairs & Black dots & Exclamation mark hairs \\
Responder group & $14 / 22(63.6 \%)$ & $17 / 22(77.3 \%)$ & $14 / 22(63.3 \%)$ & $9 / 22(40.9 \%)$ \\
Non-responder group & $9 / 17(52.9 \%)$ & $7 / 17(41.2 \%)$ & $9 / 17(52.9 \%)$ & $2 / 17(11.8 \%)$ \\
Severe side-effect group & $0 / 2$ & $2 / 2(100 \%)$ & $2 / 2(100 \%)$ & $1 / 2(50.0 \%)$ \\
Voluntary withdrawal group & $4 / 9(44.4 \%)$ & $5 / 9(55.6 \%)$ & $6 / 9(66.7 \%)$ & $3 / 9(33.3 \%)$ \\
P value & $0.339(\chi 2=3.168)$ & $0.060(\chi 2=7.424)$ & $0.678(\chi 2=1.602)$ & $0.156(\chi 2=4.843)$ \\
\hline
\end{tabular}





\section{Supplemental file}

\section{Serum Level of IL-4 Predicts Response to Topical Immunotherapy with}

\section{Diphenylcyclopropenone (DPCP) in Alopecia Areata}

Yugang Gong, Ying Zhao, Xiaoting Zhang, Shiling Qi, Shuifeng Li, Yanting Ye, Jian Yang, Sillani Caulloo, Kevin J. McElwee, Xingqi Zhang

Figure 1. Global scalp views in four cases pre- and post-treatment

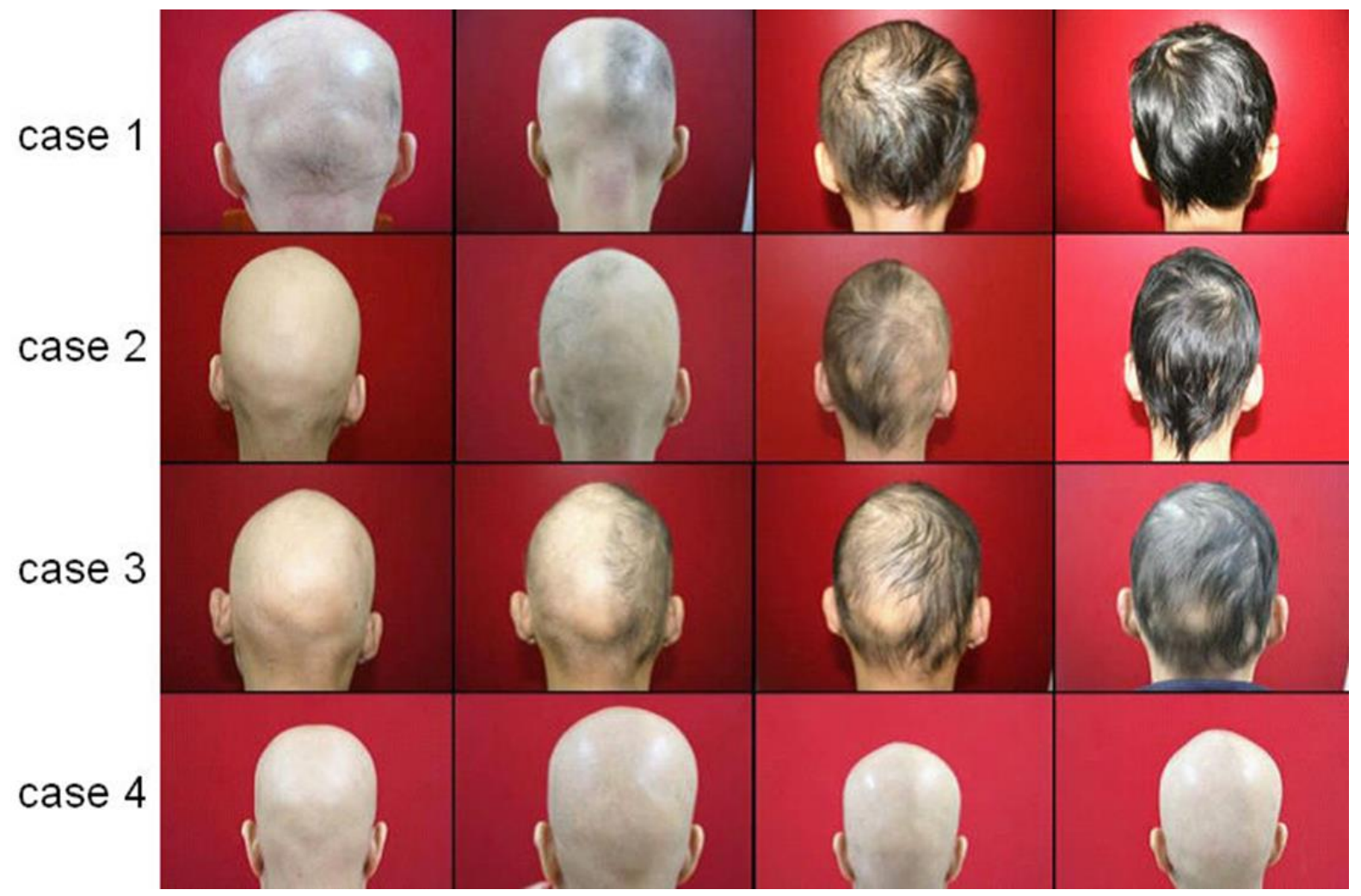

Cases 1 to 3 were in the responder group (case 1 and 2: Grade IV, case 3: Grade III), whereas case 4 was in the non-responder group. Global scalp views in four cases are shown before treatment, after 2 months treatment (case 2 for the left half scalp, other cases for the right half scalp), after 4 months treatment (case 2 on the left half scalp for 4 months and the right half 
scalp for 2 months; case 1 and 3 on the right half scalp for 4 months and the left half scalp for 2 months; case 4 on the right half scalp for 4 months), and after 6 months treatment.

\section{Figure 2. Scalp dermoscopy of AA}

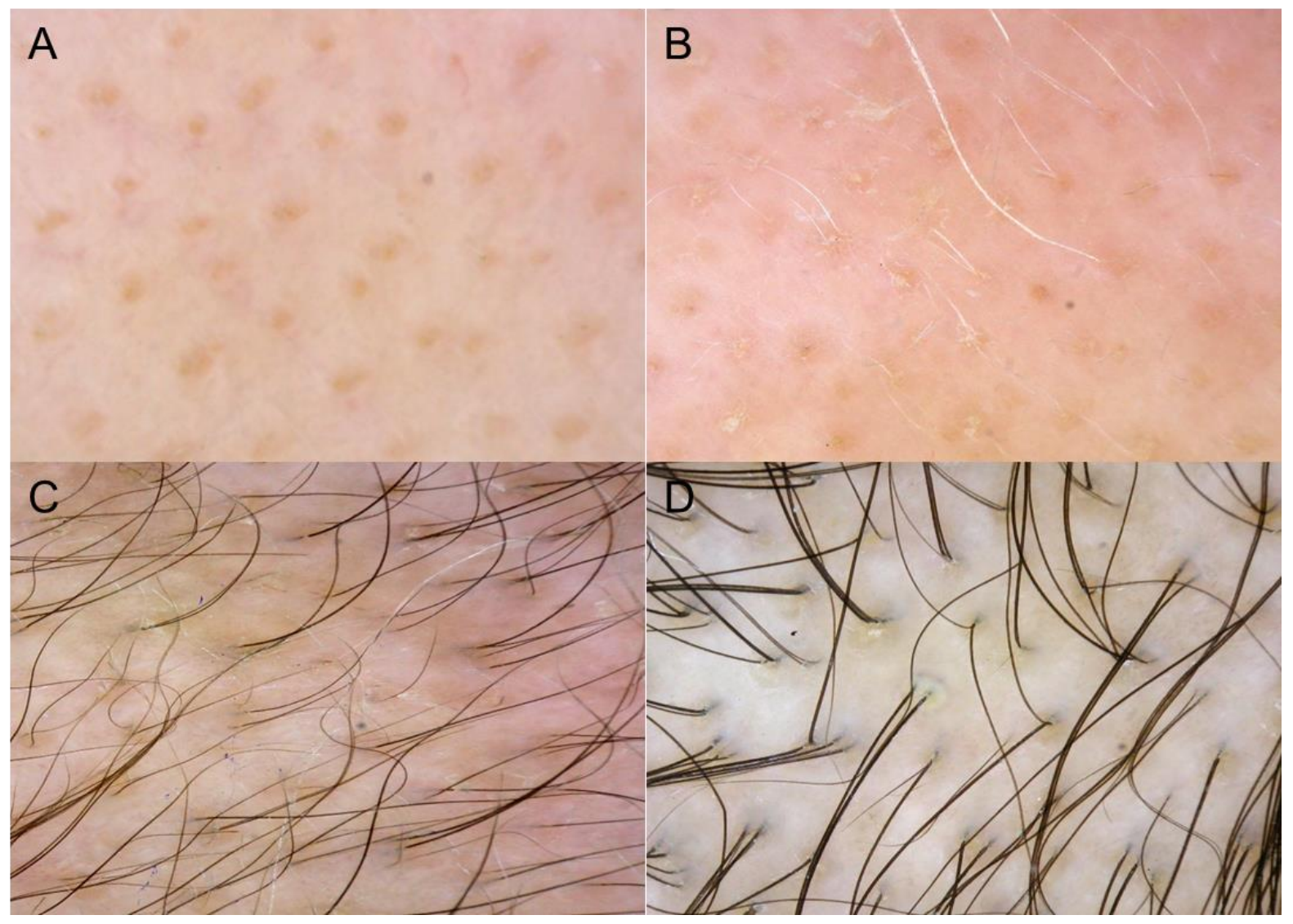

Scalp dermoscopy before treatment (A), after three (B), eight (C), and fourteen (D) weeks of DPCP treatment in the same case. 